\title{
Measurement of non-linear optical coefficients of chalcogenide glasses near the fundamental absorption band edge
}

\author{
Elena Romanova $^{1}$, Yulia Kuzyutkina ${ }^{1}$, Vladimir Shiryaev ${ }^{2}$, Nabil Abdel-Moneim ${ }^{3}$, David \\ Furniss $^{3}$, Trevor Benson ${ }^{3}$, Angela Seddon ${ }^{3}$ and Stephane Guizard ${ }^{4}$ \\ ${ }^{1}$ Saratov State University, Astrakhanskaya 83, 410012 Saratov, Russia \\ ${ }^{2}$ Institute of Chemistry of High Purity Substances of RAS, Tropinina 49, 603950 \\ Nizhny Novgorod, Russia \\ ${ }^{3}$ University of Nottingham, University Park, NG72RD Nottingham, UK \\ ${ }^{4}$ Ecole Polytechnique, 91128 Palaiseau Cedex, France \\ E-mail: elena_rmnv@yahoo.co.uk
}

\begin{abstract}
A time-resolved pump-probe method is used for the evaluation of non-linear optical coefficients of chalcogenide glasses from the As-S-Se and Ge-Se systems near their fundamental absorption band edges. The results are analyzed via comparison with the spectral dependencies of the non-linear optical coefficients of crystalline semiconductors; the role of electron transitions through the gap states of chalcogenide glasses is discussed.

OCIS codes: (190.4400) Nonlinear optics, materials; (160.2750) Glass and other amorphous materials; (320.2250) Femtosecond phenomena
\end{abstract}

\section{Introduction}

The non-linear optical response of chalcogenide glasses has been studied experimentally for the past 20 years. It was found that non-linear optical coefficients concerning to the third-order non-linear polarization were 2-3 orders greater than those of fused silica. As the optical bandgap energy $E_{\mathrm{g}}$ of a chalcogenide glass composition is usually less than $2.5 \mathrm{eV}$ [1] their fundamental absorption band (FAB) edges correspond to the bandgap wavelengths $\lambda_{\mathrm{g}}>0.5 \mu \mathrm{m}$. In fact, non-linear optical coefficients of the glass systems As-S-Se, Ge-Se, Ga-La-S and others have been measured mainly at the wavelengths $\lambda: 1.064 \mu \mathrm{m}, 1.25 \mu \mathrm{m}$ and $1.55 \mu \mathrm{m}$ of available ultrafast lasers [2]. At these wavelengths, the ratio $R_{e}=h v E_{\mathrm{g}}$ ( $h v$ is the laser photon energy) is less than 0.6. Meanwhile, the range $0.6<R_{e}<1$ is of current interest in both fundamental and applied research.

In the technology of waveguides inscription in optical glasses, the non-linear coefficients of refraction and absorption are basic parameters, which govern a high-intensity laser pulse energy deposition into a glass sample and final refractive index variation. When illuminated in a spectral range near the FAB edge, chalcogenide glasses exhibit specific photo-structural changes known as photodarkening [1]. The character of the non-linear optical response in a chalcogenide glass sample depends on whether the sample has been illuminated near to or far from the FAB edge [3].

Up to now, a theory of the non-linear optical response of non-crystalline semiconductors, in particular, chalcogenide glasses, has not been developed. In the theory of crystalline semiconductors, a two-band model $[2,4,5]$ is usually used to describe the third-order non-linear polarization. However, this model is incompatible with non-crystalline semiconductors that have energy levels in their bandgaps (so called gap states). Nevertheless the theory of crystalline semiconductors was used in some papers for evaluation of the non-linear optical coefficients of refraction $n_{2}$ and absorption $\beta_{2}$ of chalcogenide glasses [6-9]. (These coefficients correspond to the intensity-dependent parts of the refractive index $n=n_{0}+n_{2} I$ and absorption coefficient $\alpha=\alpha_{0}$ $+\beta_{2} I$, where $n_{0}$ and $\alpha_{0}$ are, respectively, the linear refractive index and single-photon absorption coefficient, and $I$ is intensity of the laser radiation.) Far from the FAB edge, when $\lambda>\lambda_{\mathrm{g}}$, comparison of the theoretical values with experimental data provides some fit [7], but there is a discrepancy near the FAB edge because in the theory of the direct-gap crystalline semiconductors, $n_{2}$ is negative-valued over some part of the spectral range $\left(R_{e}>0.7\right)$ [4]. Meanwhile in accordance with the available experimental data [2], only $n_{2}>0$ has hitherto been observed in all the range of transparency of chalcogenide glasses. 
In experiments on $n_{2}$ and $\beta_{2}$ measurements near the FAB edge, densities of photo-excited charge carriers in a sample can be large due to the single- and two- photon absorption. This effect can significantly violence the output parameters especially when laser pulses of nano- or pico- second durations are used for pumping [10].

In this work, a time-resolved pump-probe method was applied to evaluate $n_{2}$ and $\beta_{2}$ of chalcogenide glasses from the As-S-Se and Ge-Se systems near their FAB edges. In the experiment, a single femtosecond (fs) pump pulse was used for a glass sample illumination at each probe pulse delay. To avoid a cumulative effect, the sample was moved after each shot. Together with a thorough analysis of the short time scale dynamics of the non-linear optical response, this enabled us to realize the experimental conditions when the photo-excited free electrons density is small and can be neglected in a numerical model used for $n_{2}$ and $\beta_{2}$ evaluation.

\section{Experiment}

Samples of the compositions $\mathrm{As}_{40} \mathrm{~S}_{\mathrm{x}} \mathrm{Se}_{60-\mathrm{x}}(\mathrm{x}=0,15,30,45,60)$ (atomic \%) and $\mathrm{Ge}_{20} \mathrm{Se}_{80}, \mathrm{Ge}_{12} \mathrm{Se}_{88}$ were fabricated from synthesized glass rods, shaped as thin disks (thickness $\sim 1 \mathrm{~mm}$ ) and polished to a $0.25 \mu \mathrm{m}$ finish [11]. The FAB edges in absorption spectra obtained from spectrophotometric measurements $[11,12]$ are shown in Figure 1. The linear part of each curve corresponds to the Urbach tail [1]. The weak absorption tails (WAT) typically refer to the range where $\alpha_{0}<1 \mathrm{~cm}^{-1}$. These glass samples were illuminated by laser pulses (HMFW duration $\tau=50 \mathrm{fs})$ with the peak wavelength $\lambda^{p}=0.79 \mu \mathrm{m}\left(h v^{p}=1.57 \mathrm{eV}\right)$.

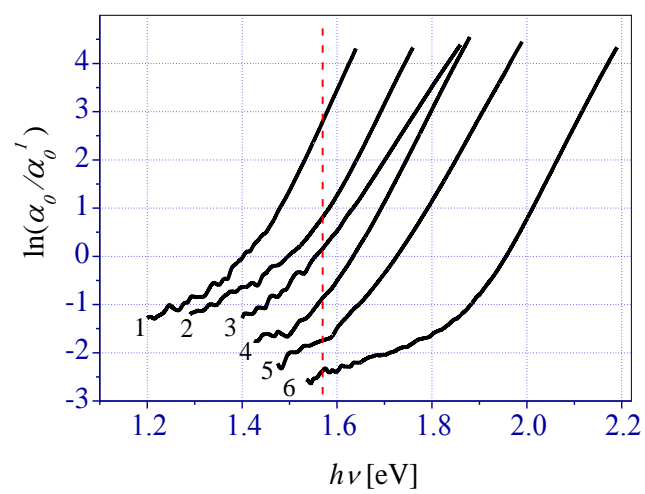

Figure 1. Logarithm of the absorption coefficient of the glass compositions $\mathrm{As}_{40} \mathrm{~S}_{\mathrm{x}} \mathrm{Se}_{60-\mathrm{x}}, \mathrm{x}=0$ (1), 15 (2), 30 (4), 45(5), 60 (6) and $\mathrm{Ge}_{20} \mathrm{Se}_{80}$ (3). The vertical dashed line indicates $h v^{p}=1.57 \mathrm{eV} \cdot \alpha_{0}^{1}=1 \mathrm{~cm}^{-1}$

Fourier Transform interferometry [13] enabled us to measure the non-linear optical response of these glass samples with femtosecond resolution in time. In the experiment, a high intensity pump pulse was focused onto a sample by a lens with a focal distance of $300 \mathrm{~mm}$ after a $3 \mathrm{~mm}$ aperture. (A schematic of the experimental set-up is shown as the inset to Figure 2). The sample was moved after each shot. A probe pulse propagating with some delay $\Delta t$ after the pump pulse was divided into two pulses at a Michelson interferometer. As the probe beam diameter was several times greater than the pump beam diameter, after the interferometer a disturbed central part of one probe beam overlapped with an unexcited peripheral part of the other one. An interference pattern was recorded at the spectrometer exit and finally a Fourier Transform was performed to extract the phase shift $\Delta \varphi$ of the probe pulse at each transverse coordinate for each $\Delta t$. The magnitude and sign of $\Delta \varphi$ induced due to the effect of cross-phase modulation were proportional to the variation of the real part of the refractive index upon the pump pulse illumination.

It was proposed in [13] that the dielectric constant of a glass sample varies due to the third-order non-linearity, free charge carriers excitation and their trapping at the gap-states. This can be observed in Figure 2, which displays the time dependence of $\Delta \varphi$ averaged over the probe beam cross-section for the sample $\mathrm{As}_{40} \mathrm{~S}_{60}$. At low pulse energy $E$ (see the curve $\Delta \varphi(\Delta t)$ at $E=0.3 \mu \mathrm{J}$ in Figure 2, filled circles), the dielectric constant changes predominantly due to the third-order non-linearity (optical Kerr effect) and this reveals itself as a positive-valued maximum in $\Delta \varphi(\Delta t)$. At higher $E$ (the curve $\Delta \varphi(\Delta t)$ at $E=9.2 \mu \mathrm{J}$ in Figure 2, open triangles), this maximum is followed by a fast $\Delta \varphi$ decrease down to negative values due to the photo-excitation of free electrons. At greater $\Delta t$, a subsequent slow increase in $\Delta \varphi$ magnitude is observed; this is assumed to be due to the charge carriers recombination (followed by a permanent change of refractive index at $\Delta t>4 \mathrm{ps}$ [3]). In chalcogenide glasses, the kinetics of charge carriers develops as both radiative and non-radiative recombination [14]. 


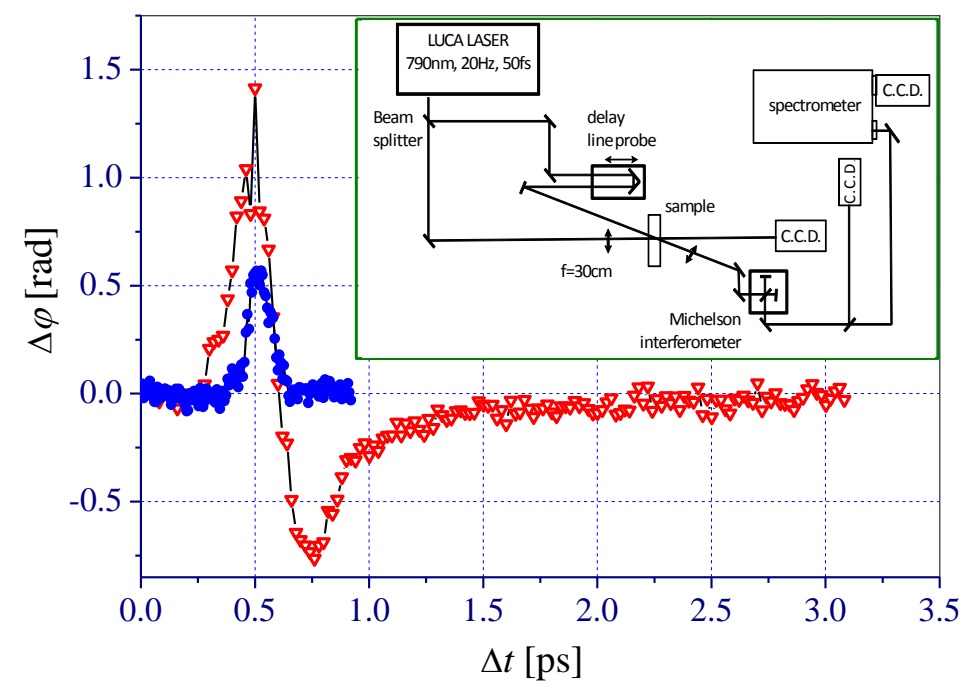

Figure 2. Time-resolved non-linear optical response of the glass samples: $\mathrm{As}_{40} \mathrm{~S}_{60}, E=0.3 \mu \mathrm{J}$ (filled circles), 9.2 $\mu \mathrm{J}$ (open triangles); inset: the pump-probe set-up scheme.

At low $E$, when the density of photo-excited electron-hole pairs is negligibly small, the propagation of the laser pulses in the sample along the $z$-direction, normal to the sample surface, is described by the equations:

$$
\begin{gathered}
\partial I_{p u} / \partial z=-\left(\alpha_{0}+\beta_{2} I_{p u}\right) I_{p u} \\
\partial I_{p r} / \partial z=-\left(\alpha_{0}+2 \beta_{2} I_{p u}\right) I_{p r} \\
\partial \varphi_{p u} / \partial z=k n_{2} I_{p u} \\
\partial \varphi_{p r} / \partial z=2 k n_{2} I_{p u}
\end{gathered}
$$

where $I_{p u}(r, z, t)$ and $I_{p r}(r, z, t), \varphi_{p u}(r, z, t)$ and $\varphi_{p r}(r, z, t)$ are, respectively, the pump and probe pulse intensities and their phases; $r$ is the transverse coordinate, $t$ is the time in the moving frame coordinate system, and $k=2 \pi \lambda^{p}$. The equations (1) do not include any derivatives with respect to the transverse coordinate $r$ because at low $E$, we have found that the laser beam spot size in the sample did not vary significantly with $\Delta t$. A small angle between the pump and probe pulses equal to $18.5^{\circ}$ in air (less than $7^{\circ}$ in a sample) was also not taken into account.

From (1), the following expressions for evaluating $n_{2}$ and $\beta_{2}$ have been obtained:

$$
\begin{gathered}
\beta_{2}=\left(1+(1-\mathrm{A})^{-1 / 2}\right) /\left(I_{p u}^{0} L^{(\alpha)}\left(1+\left(L / L_{d}\right)^{2}\right)^{-1 / 2}\right) \\
n_{2}=\Delta \varphi(L) /\left(2 k \int_{0}^{L} I_{p u}(z) d z\right)
\end{gathered}
$$

Here $I_{p u}^{0}=E \cdot \tau^{-1} \cdot\left(\pi w^{2}\right)^{-1}$ is the peak intensity of the pump pulse on the front surface of the sample, taking into account of the Fresnel reflection from the surface, $w$ is the laser beam radius, $L^{(\alpha)}=\left(1-\exp \left(-\alpha_{0} L\right)\right) / \alpha_{0}, L$ is the sample thickness. The probe pulse absorbance $\mathrm{A}=1-I_{p r}^{L} / I_{p r}^{0}$, where $I_{p r}^{L}$ and $I_{p r}^{0}$ are the peak intensities of the probe pulse at the back and front surfaces of the sample, respectively.

As the group velocity dispersion (GVD) coefficient of chalcogenide glasses is large at $\lambda^{\mathrm{p}}[1,2]$ (for $\mathrm{As}_{40} \mathrm{~S}_{60}$, the dispersion length $L_{\mathrm{d}}=0.66 \mathrm{~mm}$ that is comparable with the sample thickness $L=0.88 \mathrm{~mm}$ ) the pump intensity decrease due to the pulse broadening is to be taken into account. In derivation of (2), (3), we have done this approximately as $I_{p u}^{0}\left(1+\left(z / L_{d}\right)^{2}\right)^{-1 / 2}$.

For the evaluation of $n_{2}$ and $\beta_{2}$, the results of measurements for each sample have been analysed in order to identify the pump pulse energies when the density of the photo-excited electron-hole pairs is negligibly small (no obvious $\Delta \varphi$ decrease down to negative values, Figure.3). At these energies $(E \approx 0.2-0.3 \mu \mathrm{J})$, the transverse profiles of $\Delta \varphi$ and A (Figure 3, inset) measured at the maximums $\Delta t^{\max }$ of the $\Delta \varphi(\Delta t)$ curves have been approximated by Gaussian functions and the mean values of $\Delta \varphi$ and $\mathrm{A}$ at the beam axis have been defined. 


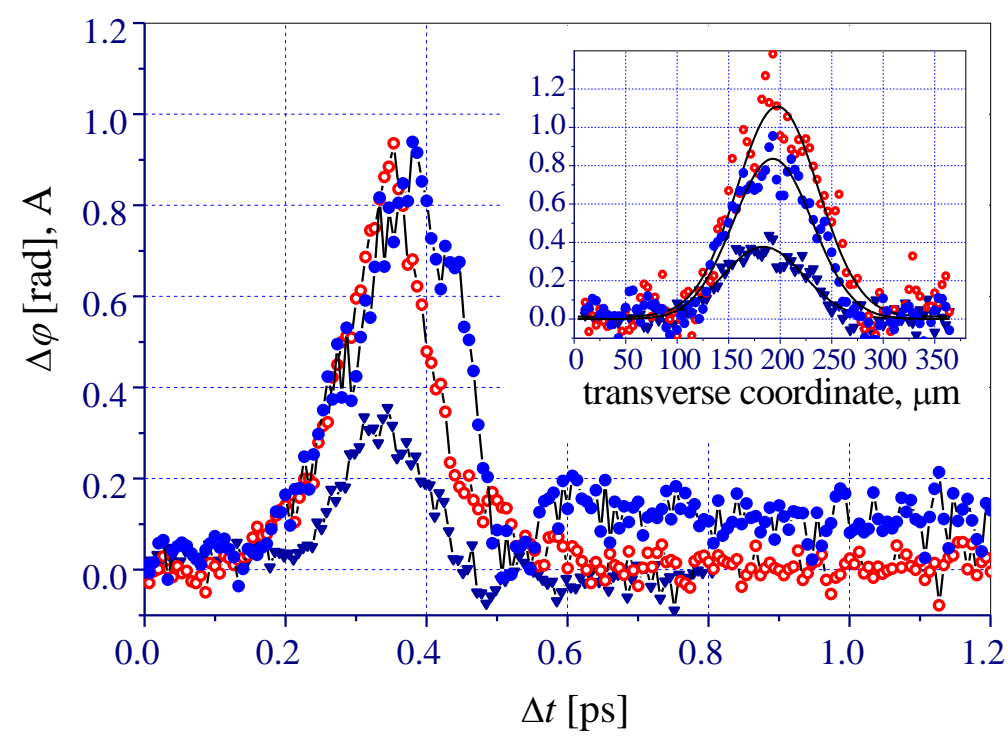

Figure 3. Time-resolved non-linear optical response of the glass samples: $\mathrm{As}_{40} \mathrm{~S}_{30} \mathrm{Se}_{30}$ (filled triangles), $\mathrm{Ge}_{20} \mathrm{Se}_{80}$ (open circles - $\Delta \varphi$, filled circles - A), $E=0.3 \mu \mathrm{J}$; inset: transverse profiles of $\Delta \varphi$ and $\mathrm{A}$ at $\Delta t=\Delta t^{\max }$

\section{Results}

As the $\beta_{2}$ magnitudes of $\mathrm{As}_{40} \mathrm{~S}_{60}$ at $\lambda=0.8 \mu \mathrm{m}$ have been measured in $[15,16]$ by using femtosecond laser pulses, these results $\left(\beta_{2} \approx 2 \mathrm{~cm} / \mathrm{GW}\right)$ have been taken in this work for comparison. As is known from literature [10], using of the nano- or pico- second laser pulses provided greater magnitudes: $\beta_{2} \approx 3-10 \mathrm{~cm} / \mathrm{GW}$. As follows from Figure 2, densities of the charge carriers at large $E$ are large and this can violence the results of $n_{2}$ measurements with the long pulses. The increase of $\beta_{2}$ and $n_{2}$ magnitudes with the pump pulse duration due to thermal effects has been demonstrated in [17].

For $\mathrm{As}_{40} \mathrm{~S}_{60}$ composition, a series of measurements was undertaken in order to evaluate mean $\beta_{2}$ and $n_{2}$ magnitudes (table 1) by using (2), (3). Other parameters in table 1 have been obtained using spectrophotometric measurements [12]. $E_{g}$ has been evaluated as an iso - absorption gap at $\alpha_{0}=10^{3} \mathrm{~cm}^{-1}$. For the system As-S-Se, the obtained $E_{g}$ magnitudes are in a good agreement with the results published in [18].

The same technique has been applied to other glass samples and the results are collected in table 1. Only positive values of $n_{2}$ have been obtained experimentally. At $E=0.3 \mu \mathrm{J}$, the magnitude of $I_{p u}^{0}$ for all the samples was around $20-25 \mathrm{GW} / \mathrm{cm}^{2}$. The non-linear part $\beta_{2} I_{p u}^{0}$ of the absorption coefficient varied in the range $60-90$ $\mathrm{cm}^{-1}$ that is much greater than $\alpha_{0}$ (table 1) in (1a), (1b). Then the curve $\mathrm{A}(\Delta t)$ at $E=0.3 \mu \mathrm{J}$ in Figure 3 is related mainly to the two-photon absorption in $\mathrm{Ge}_{20} \mathrm{Se}_{80}$. A weak absorption by free electrons reveals itself as a small shift of the curve maximum with respect to the maximum of $\Delta \varphi(\Delta t)$. Some residual absorption at $\Delta t>0.6 \mathrm{ps}$ is due to the electrons transitions to the gap states [13]. Because of the large $\beta_{2} I_{p u}^{0}$, the non-linear optical response develops at the front sample surface over a distance of less than $0.1 \mathrm{~mm}$.

Table 1. Optical parameters defined at $\lambda^{\mathrm{p}}(0.79 \mu \mathrm{m})$

\begin{tabular}{ccccccc}
\hline Composition & $E_{\mathrm{g}}, \mathrm{eV}$ & $R_{\mathrm{e}}$ & $n_{0}$ & $\alpha_{0}\left(\mathrm{~cm}^{-1}\right)$ & $\beta_{2}\left(\mathrm{~cm} \mathrm{GW}^{-1}\right)$ & $n_{2} \bullet 10^{14}\left(\mathrm{~cm}^{2} \mathrm{~W}^{-1}\right)$ \\
\hline $\mathrm{As}_{40} \mathrm{~S}_{60}$ & 2.35 & 0.66 & 2.52 & 0.1 & 2.0 & 0.8 \\
$\mathrm{As}_{40} \mathrm{~S}_{45} \mathrm{Se}_{15}$ & 2.20 & 0.71 & 2.65 & 0.2 & 2.2 & 0.4 \\
$\mathrm{As}_{40} \mathrm{~S}_{30} \mathrm{Se}_{30}$ & 2.00 & 0.78 & 2.80 & 0.4 & 2.7 & 0.4 \\
$\mathrm{As}_{40} \mathrm{~S}_{15} \mathrm{Se}_{45}$ & 1.90 & 0.82 & 2.95 & 2.2 & 4.0 & 0.6 \\
$\mathrm{Ge}_{20} \mathrm{Se}_{80}$ & 2.03 & 0.77 & 2.52 & 1.2 & 3.9 & 1.3 \\
$\mathrm{Ge}_{12} \mathrm{Se}_{88}$ & 1.95 & 0.80 & 2.68 & 3.5 & 3.9 & 0.9 \\
\hline
\end{tabular}




\section{Discussion}

As comparative experimental data at $\lambda^{\mathrm{p}}$ are currently not available in literature, we have used the theory of the non-linear optical response of the crystalline semiconductors in order to analyze these results, similar to the approach used in [6-9]. The dependencies $\beta_{2}(h v), n_{2}(h v)$ for direct-gap and indirect-gap crystalline semiconductors were obtained in [4] and [5], respectively, by using the non-linear Kramers-Kronig relation. For the direct-gap crystalline semiconductors,

$$
n_{2}(h v)=n_{2}^{0} \cdot F_{1}(h v)
$$

with the spectral function [4]:

$$
F_{1}(x)=K_{1} \cdot\left(6 x-3 x^{2}-x^{3}-\frac{3}{4} x^{4}-\frac{3}{4} x^{5}-2+2(1-2 x)^{3 / 2} \Theta(1-2 x)\right) \cdot(2 x)^{-6} \cdot\left(n_{0}^{0} E_{g}^{2}\right)^{-2}
$$

where $x=h v / E_{g}$. In (5), $K_{1}=K \cdot \hbar c \cdot E_{p}^{1 / 2} / 2, K$ is a constant and $E_{\mathrm{p}}$ is related to the interband momentum matrix element that is nearly material independent [4], $\Theta(t)=1(t \geq 0), 0(t<0)$. The magnitude of $n_{2}$ in the low frequency limit $n_{2}^{0}=n_{2}(\nu \rightarrow 0)$ has been expressed by Lines [19] in terms of the Sellmeier gap energy $E_{\mathrm{S}}$ :

$$
n_{2}^{0}=Q \cdot d_{b}^{2}\left(\left(n_{0}^{0}\right)^{2}+2\right)^{3}\left(\left(n_{0}^{0}\right)^{2}-1\right)\left(n_{0}^{0} \cdot E_{S}\right)^{-2},\left[\mathrm{~cm}^{2} / W\right]
$$

where $d_{b}[\mathrm{~nm}]$ is a mean length of the cation-anion link, and $n_{0}^{0}$ is the linear refractive index in the low frequency limit. For chalcogenide glasses, $E_{\mathrm{S}} \approx 2.5 \cdot E_{\mathrm{g}}[19]$. The coefficient $Q$ in (6) is a constant, which depends on a reference standard, i.e. a material for which $n_{2}$ values have been precisely measured. In [19], $Q=1.7 \times 10^{-14}$ was obtained in theory, but in order to fit the reference standard for $\mathrm{CS}_{2}$ at $\lambda=1 \mu \mathrm{m}$, the $Q$ magnitude was taken as twice greater than this, i.e. as $3.4 \times 10^{-14}$.

The spectral dependence of the two-photon absorption coefficient:

$$
\beta_{2}(h v)=K_{2} \cdot F_{2}(h v)
$$

where the spectral function from [4] defined in the range $h v>E_{g} / 2$ is:

$$
F_{2}(x)=(2 x-1)^{3 / 2} \cdot(2 x)^{-5} \cdot\left(n_{0}^{0} E_{g}^{3 / 2}\right)^{-2}
$$

and $K_{2}=K \cdot E_{p}^{1 / 2}$. The magnitudes of $n_{0}^{0}$ have been evaluated by using the Moss rule: $n_{0}^{0}=3 / E_{g}^{1 / 4}$, where $E_{\mathrm{g}}$ is in units of $\mathrm{eV}$ [7].

For comparison with the obtained experimental data, spectral dependencies $n_{2}(\lambda)$ have been calculated by using (4) - (6) and are plotted in Figure 4a. The coefficient $K_{1}$ in (5) has been evaluated for each glass composition from the condition: $F_{l}(h v) \rightarrow 1$ when $v \rightarrow 0$, which fits (4). It was found that the experimental $n_{2}$ magnitudes available in the literature for $\mathrm{As}_{40} \mathrm{~S}_{60}$ (open circles in Figure $4 \mathrm{a}$ at $\lambda>1 \mu \mathrm{m}$ ) are located between the curves $n_{2}(\lambda)$ plotted with $Q=1.7 \cdot 10^{-14}$ (solid line 1 ) and $Q=3.4 \cdot 10^{-14}$ (dashed line 1). Such a large variation of the measured $n_{2}$ values available in literature is likely to be due to the use of different laser pulse durations in the experimental set-ups, as has been mentioned above.

Similarly, we have plotted such curves for $\mathrm{As}_{40} \mathrm{~S}_{30} \mathrm{Se}_{30}$ (solid and dashed lines 2 in Figure $4 \mathrm{a}$ ). As $E_{\mathrm{g}}$ of the composition $\mathrm{Ge}_{20} \mathrm{Se}_{80}$ is almost the same as that of $\mathrm{As}_{40} \mathrm{~S}_{30} \mathrm{Se}_{30}$ their calculated spectral curves are almost identical and they are plotted together on Figure 4a. Experimental data available for these samples are in an agreement with the curves $n_{2}(\lambda)$ at $\lambda>1 \mu \mathrm{m}$ within the experimental error $15-20 \%$ that is typical for measurements of the non-linear optical coefficients [2].

The spectral dependencies $\beta_{2}(\lambda)$ of the glass samples of the system As-S-Se have been calculated by using (7), (8) and are plotted in Figure 4b. The coefficient $K_{2}$ has been evaluated from the available data for As40 $\mathrm{S}_{60}$ : $\beta_{2}(\lambda) \approx 2 \mathrm{~cm} / \mathrm{GW}$ at $\lambda=0.8 \mu \mathrm{m}[15,16]$. For the sample $\mathrm{Ge}_{20} \mathrm{Se}_{80}, K_{2}$ estimation was based on the result from [20]: $\beta_{2}(\lambda)=1.8 \mathrm{~cm} / \mathrm{GW}$ at $\lambda=1.064 \mu \mathrm{m}$. In fact, there is little published experimental data for comparison. For crystalline semiconductors, $\beta_{2}(\lambda)=0$ in the range $\lambda>2 \lambda_{\mathrm{g}}$. However for chalcogenide glasses, there are non-zero literature data in this range $[2,18-24]$ as shown in Figure $4 \mathrm{~b}$. At these points, the non-linear absorption coefficient can be attributed to consecutive electron transitions involving the gap states (two-step transitions) [21]. 

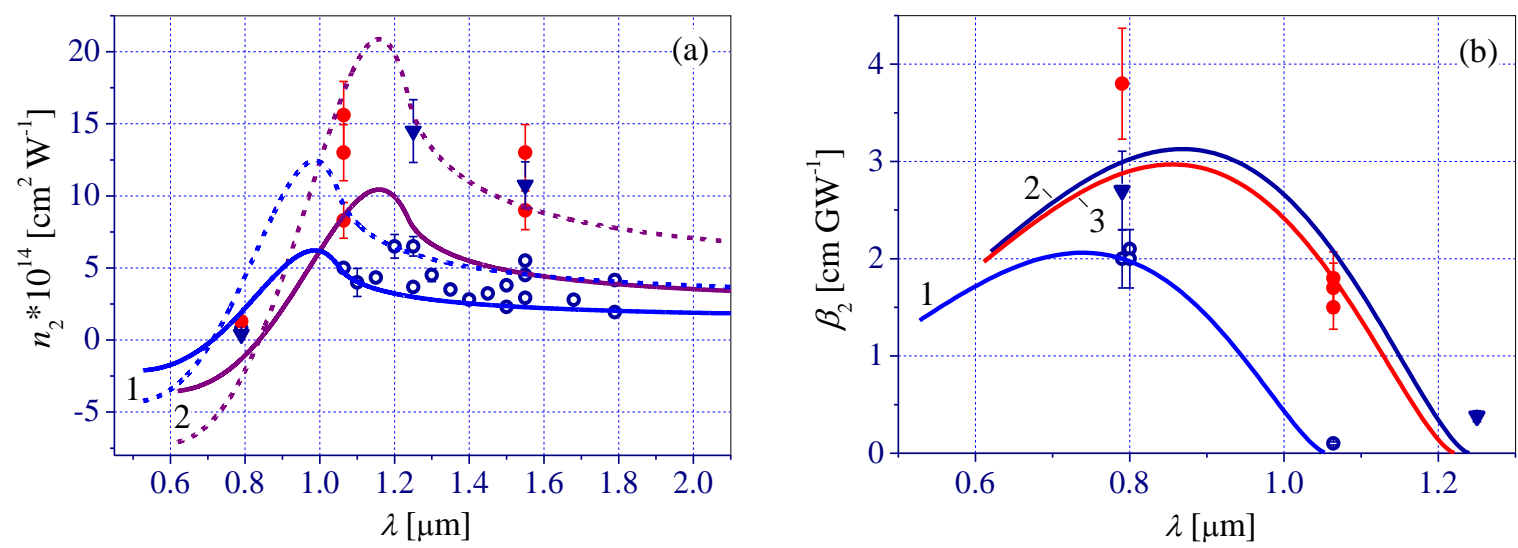

Figure 4. Experimental data from the present work at $\lambda^{p}=0.79 \mu \mathrm{m}$ and from [2,18-24] (symbols). Calculated wavelength dependencies of: a) $n_{2}\left(Q=1.7 \cdot 10^{-14}\right.$ (solid lines), 3.4·10-14 (dashed lines)); 1 - As $40 \mathrm{~S}_{60}$ (open circles), 2 - $\mathrm{As}_{40} \mathrm{~S}_{30} \mathrm{Se}_{30}$ (filled triangles) and $\mathrm{Ge}_{20} \mathrm{Se}_{80}$ (filled circles). b) $\beta_{2}, 1$ - $\mathrm{As}_{40} \mathrm{~S}_{60}$ (open circles), 2 - $\mathrm{As}_{40} \mathrm{~S}_{30} \mathrm{Se}_{30}$ (filled triangles), $3-\mathrm{Ge}_{20} \mathrm{Se}_{80}$ (filled circles)

As $E_{\mathrm{g}}$ is actually the main decisive parameter in the theory of the non-linear optical response of crystalline semiconductors, it is reasonable to analyze its role in the non-linear optical response of chalcogenide glasses. From the literature data shown in Figure $4 \mathrm{a}$, it can be seen that at a given wavelength $\lambda>1 \mu \mathrm{m}, n_{2}$ is greater for the compositions with smaller $E_{\mathrm{g}}$ (table 1), as is already known [2]. Some agreement is also noticeable with the spectral dependencies of the direct-gap crystalline semiconductors (lines in Figure 4a). However near the FAB edge, the positive-valued $n_{2}$ measured in the present work (data at $\lambda^{\mathrm{p}}=0.79 \mu \mathrm{m}$ in Figure 4a, taken from table 1) do not fit these dependencies, which have $n_{2}<0$ in the range $R_{e}>0.7$, for some compositions, namely $\mathrm{As}_{40} \mathrm{~S}_{30} \mathrm{Se}_{30}$ and $\mathrm{Ge}_{20} \mathrm{Se}_{80}$. Far from the FAB edge, these compositions having approximately the same $E_{\mathrm{g}}(\approx 2$ $\mathrm{eV}$, table 1) exhibit similar $n_{2}$ values (filled circles and triangles in Figure $4 \mathrm{a}$ at $\lambda>1 \mu \mathrm{m}$ ) within the experimental error. Near the FAB edge at $\lambda^{p}, n_{2}$ and $\beta_{2}$ of the $\mathrm{Ge}_{20} \mathrm{Se}_{80}$ sample are much larger than those measured for the $\mathrm{As}_{40} \mathrm{~S}_{30} \mathrm{Se}_{30}$ sample (Table 1).

In Figure 1, we can see that the sample $\mathrm{Ge}_{20} \mathrm{Se}_{80}$ was illuminated in the range of its Urbach tail $\left(\ln \left(\alpha_{0} / \alpha_{0}^{1}\right)>0\right)$, while the sample $\mathrm{As}_{40} \mathrm{~S}_{30} \mathrm{Se}_{30}$ - in the range of its WAT tail $\left(\ln \left(\alpha_{0} / \alpha_{0}^{1}\right)<0\right)$. The sample $\mathrm{As}_{40} \mathrm{~S}_{15} \mathrm{Se}_{45}$ illuminated at its Urbach tail has the magnitude of $\beta_{2}$ comparable with that measured here for $\mathrm{Ge}_{20} \mathrm{Se}_{80}$ (table 1). As the Urbach tail is associated with the photo-induced electron transitions involving the gap states [1,2], we can assume that probabilities of the two-step transitions through the real states in the bandgap are greater than for the two-photon transitions through virtual states as assumed in the two-band models of the crystalline semiconductors. If a sample is illuminated in the range of its WAT tail, the two-photon transitions should happen mainly through the virtual states because the density of gap states involved into these transitions is small. In Figure 1, this occurs for three samples of the composition $\mathrm{As}_{40} \mathrm{~S}_{\mathrm{x}} \mathrm{Se}_{60-\mathrm{x}}(\mathrm{x}=30,45,60)$. In the range of the Urbach tail, the probabilities of the two-step transitions through the gap states may increase. This explains why the $\beta_{2}$ magnitude of the sample $\mathrm{Ge}_{20} \mathrm{Se}_{80}$ measured in the present work (filled circle at $\lambda^{\mathrm{p}}$ in Figure $4 \mathrm{~b}$ ) is greater than the one calculated at $\lambda^{p}$ by using (7), (8) (line 3 in Figure 4b). Similarly for the sample $\mathrm{As}_{40} \mathrm{~S}_{15} \mathrm{Se}_{45}$, the $\beta_{2}$ magnitude measured and presented in table 1 is greater than the value of 3.2 $\mathrm{cm} / \mathrm{GW}$ calculated at $\lambda^{\mathrm{p}}$ by using (7), (8).

\section{Conclusions}

In conclusion, Fourier Transform interferometry with the femtosecond resolution in time has been used to measure the nonlinear optical response of a variety of chalcogenide glasses of the systems As$\mathrm{S}-\mathrm{Se}$ and $\mathrm{Ge}-\mathrm{Se}$ and evaluate their non-linear optical coefficients of refraction and absorption near the FAB edge. The technique has been based on the thorough analysis of the results of measurements at different pump pulse energies and identification of the conditions when the nonlinear optical response is defined predominantly by the optical Kerr effect. In fact, this effect driven by the electronic part of a polarization is much faster than structural transformations revealing in a permanent increase of 
refractive index of a glass sample in a long-time scale dynamics of the non-linear optical response [3]. This separation in time of different contributing effects enabled us to have got the reliable and reproducible magnitudes of the non-linear optical coefficients near the FAB edge.

Comparison with the results of the theory of crystalline semiconductors has revealed that spectral dependence $\beta_{2}(h v)$ of the direct-gap crystalline semiconductors exhibit an agreement (within an experimental error) with the available literature data and our results for the glass samples illuminated at their WAT tails. For the samples illuminated at their Urbach tails, when electron transitions through the gap states are possible, the magnitudes of $\beta_{2}$ differ substantially from that calculated by using the results of the theory of the direct-gap crystalline semiconductors.

Our results of $n_{2}$ evaluation and the data available in literature reveal that $n_{2}$ magnitudes are always positive-valued that is different from the direct-gap crystalline semiconductors having negative-valued $n_{2}$ in some spectral range near the FAB edge. We can conclude that a shape of the spectral dependencies $n_{2}(h v)$ of chalcogenide glasses near the FAB edge is still unknown and needs further investigation.

Acknowledgments This research was supported by the LaserLab-Europe network (the project ref. number SLIC001675), the Royal Society (IJP-2010/R1, IES-2013/R2) and by the Russian Science Foundation (Grant No. 16-13-10251).

\section{References}

[1] M.A. Popescu, Non - Crystalline Chalcogenides, Kluwer Acad. Publishers, New York, Boston, Dordrecht, London, Moscow, 2002.

[2] A. Zakery and S.R. Elliot, Optical nonlinearities in chalcogenide glasses and their applications, Springer-Velag, Berlin, Heidelberg, New York, 2007.

[3] E.A. Romanova, Yu.S. Kuzyutkina, A.I..Konyukhov, N..Abdel-Moneim, A.B. Seddon, T.M. Benson, S. Guizard, A. Mouskeftas, Nonlinear optical response and heating of chalcogenide glasses upon irradiation by the ultrashort laser pulses, Opt. Eng. 53 (2014) 071812 DOI: 10.1117/1.OE.53.7.071812

[4] M. Sheik-Bahae, D.J. Hagan, and E.W. Van Stryland, Dispersion and band-gap scaling of the electronic Kerr effect in solids associated with two-photon absorption, Phys. Rev. Lett. 65 (1990) 96 DOI:https://doi.org/10.1103/PhysRevLett.65.96

[5] M. Dinu, Dispersion of phonon-assisted nonresonant third-order nonlinearities, IEEE J. Quantum Electron. 39 (2003) 1498 DOI: 10.1109/JQE.2003.818277

[6] G. Lenz, J. Zimmermann, T. Katsufuji, M.E. Lines, H.Y. Hwang, S. Spalter, R.E. Slusher, S.W. Cheong, J.S. Sanghera, I.D. Aggarwal, Large Kerr effect in bulk Se-based chalcogenide glasses, Opt. Lett. 25 (2000) 254 DOI: https: //doi.org/ 10.1364/OL.25.000254

[7] K. Tanaka, Nonlinear optics in glasses: How can we analyze?, J. Phys. Chem. Solids 68 (2007) 896 DOI: http://dx.doi.org/10.1016/j.jpcs.2006.11.035

[8] R. Todorov, J. Tasseva, T. Babeva, Thin chalcogenide films for photonic applications, in: A. Massaro (Ed.), Photonic crystals-innovative systems, lasers and waveguides, In Tech, 2012, pp. 143-168.

[9] T. Wang, X. Gai, W. Wei, R. Wang, Z. Yang, X. Shen, S. Madden, and B. Luther-Davies, Systematic z-scan measurements of the third-order nonlinearity of chalcogenide glasses, Opt. Mater. Express 4 (2014) 1011 DOI: https://doi.org/10.1364/OME.4.001011

[10] K. Tanaka, Two-photon absorption spectroscopy of $\mathrm{As}_{2} \mathrm{~S}_{3}$ glass, Appl. Phys. Lett. 80 (2002) 177 DOI: http://doi.org/10.1063/1.1433908

[11] N. Abdel-Moneim, Fabrication of planar optical components in chalcogenide glasses $(\mathrm{PhD}$ Thesis), University of Nottingham, 2013.

[12] Yu.S. Kuzyutkina, E.A. Romanova, V.I. Kocubey, V.S. Shiryaev, Specific features of linear and nonlinear optical responses of chalcogenide glasses in the As-S-Se and As-Se-Te systems, Opt. Spectrosc. 117 (2014) 49, doi:10.1134/S0030400X14070121

[13] P. Martin, S. Guizard, Ph. Daguzan, G. Petite, P. D’Oliveira, P. Meynadier, M. Perdrix, Subpicosecond study of carrier trapping dynamics in wide-band-gap crystals, Phys. Rev. B 55 (1997) 5799 DOI:https://doi.org/10.1103/PhysRevB.55.5799 
[14] R.A. Street, Luminescence in amorphous semiconductors, Adv. Phys. 25 (1976) 397 DOI: http://dx.doi.org/10.1080/00018737600101412

[15] S. Juodkazis, T. Kondo, H. Misawa, Photo-structuring of $\mathrm{As}_{2} \mathrm{~S}_{3}$ glass by femtosecond irradiation, Opt. Express 14 (2006) 7751 DOI: https://doi.org/ 10.1364/OE.14.007751

[16] I. Blonskyi, V. Kadan, O. Shpotyuk, M. Iovu, I. Pavlov, Femtosecond filamentation in chalcogenide glasses limited by two-photon absorption, Opt. Mater. 32 (2010) 1553 DOI: http://dx.doi.org/10.1016/j.optmat.2010.07.006

[17] K. Shinkawa and K. Ogusu, Pulse-width dependence of optical nonlinearities in As2Se3 chalcogenide glass in the picosecond-to-nanosecond region, Opt. Expr. 16 (2008) 18230 DOI: https://doi.org/10.1364/ OE.16.018230

[18] J.S. Sanghera, L.B. Shaw, P. Pureza, V.Q. Nguyen, D. Gibson, L. Busse, and I.D. Aggarwal, Nonlinear properties of chalcogenide glass fibers, Int. J. Appl. Glass Sci. 1 (2010) 296 DOI: 10.1111/j.2041-1294.2010.00021.x

[19] M.E. Lines, Oxide glasses for fast photonic switching: A comparative study, J. Appl. Phys. 69 (1991) 6876 DOI: http://dx.doi.org/10.1063/1.347677

[20] J. Troles, S. Fmektala, G. Boudebs, A. Monteil, B. Bureau, J. Lucas, Optical limiting behavior of infrared chalcogenide glass, J. Optoelectron. Adv. Mater. 4 (2002) 729

[21] K. Tanaka, Two-photon optical absorption in amorphous materials, J. Non-Crystal. Solids 338340 (2004) 534 DOI: 10.1016/j.jnoncrysol.2004.03.036

[22] J. Troles, S. Fmektala, M. Guignard, P. Houizot, V. Nazabal, H. Zeghlache, G. Boudebs, V. Couderc, Third and second order non linear optical properties of chalcogenide glasses on bulk and fibers, in: Proc. Int. Conf. on Transparent Optical Networks, 2 (2005) 242 DOI: 10.1109/ICTON.2005.1506143

[23] J. M. Harbold, F. Ö. Ilday, F. W. Wise, J. S. Sanghera, V. Q. Nguyen, L. B. Shaw, and I. D. Aggarwal, Highly nonlinear As-S-Se glasses for all-optical switching, Opt. Lett. 27 (2002) 119 DOI: https://doi.org/10.1364/OL.27.000119

[24] L. Zhang, Y. Yue, Y. Xiao-Li, J. Wang, R. G. Beausoleil, A.E. Willner, Flat and low dispersion in highly nonlinear slot waveguides, Opt. Express 18 (2010) 13187 DOI: https://doi.org/ 10.1364/OE.18.013187

\section{Figure captions}

Figure 1.

Logarithm of the absorption coefficient of the glass compositions $\mathrm{As}_{40} \mathrm{~S}_{\mathrm{x}} \mathrm{Se}_{60-\mathrm{x}}, \mathrm{x}=0$ (1), 15 (2), 30 (4), 45(5), 60 (6) and $\mathrm{Ge}_{20} \mathrm{Se}_{80}$ (3). The vertical dashed line indicates $h v^{p}=1.57 \mathrm{eV} \cdot \alpha_{0}^{1}=1 \mathrm{~cm}^{-1}$

Figure 2.

Time-resolved non-linear optical response of the glass samples: $\mathrm{As}_{40} \mathrm{~S}_{60}, E=0.3 \mu \mathrm{J}$ (filled circles), 9.2 $\mu \mathrm{J}$ (open triangles); inset: the pump-probe set-up scheme.

Figure 3.

Time-resolved non-linear optical response of the glass samples: $\mathrm{As}_{40} \mathrm{~S}_{30} \mathrm{Se}_{30}$ (filled triangles), $\mathrm{Ge}_{20} \mathrm{Se}_{80}$ (open circles - $\Delta \varphi$, filled circles - A), $E=0.3 \mu \mathrm{J}$; inset: transverse profiles of $\Delta \varphi$ and A at $\Delta t=\Delta t^{\max }$.

Figure 4.

Experimental data from the present work at $\lambda^{\mathrm{p}}=0.79 \mu \mathrm{m}$ and from [2,18-24] (symbols). Calculated wavelength dependencies of: a) $n_{2}\left(Q=1.7 \cdot 10^{-14}\right.$ (solid lines), $3.4 \cdot 10^{-14}$ (dashed lines)); 1 - $\mathrm{As}_{40} \mathrm{~S}_{60}$ (open circles), 2 - $\mathrm{As}_{40} \mathrm{~S}_{30} \mathrm{Se}_{30}$ (filled triangles) and $\mathrm{Ge}_{20} \mathrm{Se}_{80}$ (filled circles). b) $\beta_{2}, 1$ - $\mathrm{As}_{40} \mathrm{~S}_{60}$ (open circles), 2 - $\mathrm{As}_{40} \mathrm{~S}_{30} \mathrm{Se}_{30}$ (filled triangles), 3 - $\mathrm{Ge}_{20} \mathrm{Se}_{80}$ (filled circles) 